\title{
A Degenerative Intraspinal Cyst Mimicking a Nerve Root: A Case Report on an Intraoperative Challenge
}

\author{
Hyeun Sung Kim ${ }^{1}$ Ravindra Singh ${ }^{1}$, Nitin Maruti Adsul', Osama Nezar Kashlan ${ }^{3}$, Bong Choon Choi ${ }^{4}$, Sung Woon $\mathrm{Oh}^{1}$, \\ Jung Hoon Noh', Chang Hwan Park ${ }^{5}$, Il Tae Jang ${ }^{1}$, Seong Hoon $\mathrm{Oh}^{6}$ \\ ${ }^{1}$ Department of Neurosurgery, Nanoori Gangnam Hospital, Seoul, Korea, ${ }^{2}$ Department of Ortho-spine Surgery, Sir Ganga Ram Hospital, \\ Delhi, India, ${ }^{3}$ Department of Neurosurgery, University of Michigan, USA, ${ }^{4}$ Department of Pain Center, Seiyeon Pain Clinic, Seoul, Korea, \\ ${ }^{5}$ Medical School, University of Debrecen, Hungary, ' Department of Neurosurgery, Nanoori Incheon Hospital, Incheon, Korea
}

Corresponding Author: Hyeun Sung Kim, MD, PhD Department of Neurosurgery, Nanoori Hospital, 731, Eonju-ro, Gangnam-gu, Seoul 06048, Korea

Tel: $+82-2-6003-9767$

Fax: +82-2-3445-9755

E-mail: neurospinekim@gmail.com

Received: December 3, 2018

Revised: May 4, 2019

Accepted: May 14, 2019

\begin{abstract}
Various intraspinal cysts have been described in the literature. Sometimes these cysts are difficult to recognize intraoperative and can place a surgeon in dilemma. We report a case of a degenerative intraspinal cyst with severe adhesion with dura, which was mimicking as a nerve root and posed a diagnostic dilemma during surgery. A Sixty-year-old man presented with insidious onset, gradually progressing lower back pain, right leg pain and neurological claudication of six months duration. The pain radiated to the right S1 dermatome. Right side straight leg raise test was positive at $45^{\circ}$. Sensations were diminished over the right L5 and S1 dermatomes. Motor function was normal. MRI showed a large cystic lesion at right L5-S1 level. The cyst appeared to compress the dural sac and traversing right S1 root at L5-S1 level. The lesion was isointense on T1-weighted image and hyperintense on T2-weighted image. While treating this condition using the uniportal full endoscopic technique the cyst appeared as nerve root. Meticulous dissection was required to separate the cyst from neural structures. Histology confirmed the diagnosis of a degenerative intraspinal cyst. The patient had significant improvement after surgery and at six months follow up he was completely asymptomatic. Various cysts can occur in the intraspinal canal, and careful attention should be paid to minimize the nerve injury in the presence of severe adhesions.
\end{abstract}

Key Words: Cysts, Spinal nerve root, Dura, Adhesions, Endoscopy

\section{INTRODUCTION}

Many case reports of various types of degenerative cysts are available ${ }^{3,47)}$. These cysts can surprise surgeons with their appearance and place a diagnostic challenge both preoperatively and intraoperatively. We report a case of degenerative intraspinal cyst with severe adhesion to dura, which mimicked a nerve root and created a diagnostic dilemma during surgery.

\section{CASE DESCRIPTION}

A 60-year-old man presented to our clinic with insidious onset, gradually progressive lower back pain, right leg pain and neurological claudication of six months duration. The pain was radiating to the right S1 dermatome. Visual analogue score (VAS) for pain was 8 .

On examination, patient had a positive straight leg raise test at 45 degrees on the right side. His sensation diminished on the right $\mathrm{L} 5$ and S1 dermatomes. However, the patient had no motor weakness.

Magnetic resonance imaging (MRI) of the patient showed a large cystic lesion at right L5-S1 level. The cyst was compressing the dural sac and traversing right S1 root at L5-S1 level. The lesion was isointense on T1-weighted images and hyperintense on T2-weighted images (Fig. 1).
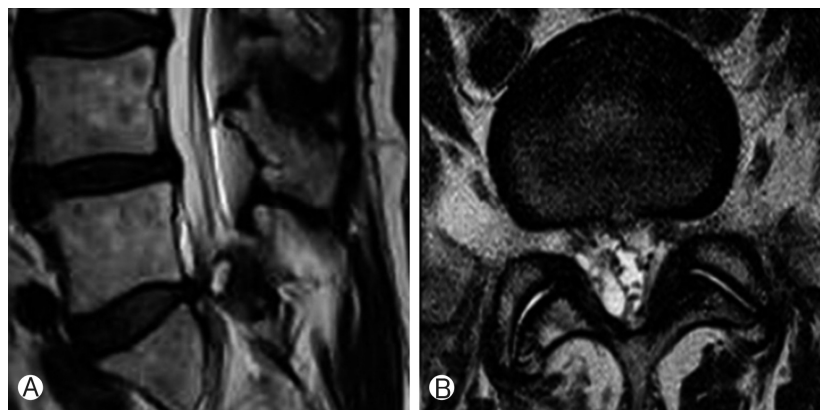

Fig. 1. Pre-operative MRI image showing cystic lesion in sagittal (A) and (B) axial images. 

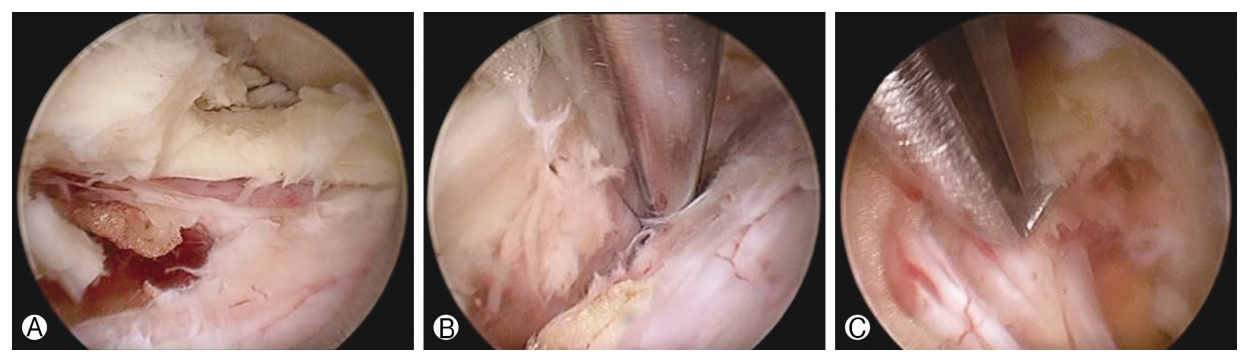

Fig. 2. Intra-operative endoscopic images showing (A) showing severe adhesions, (B) Dissection through adhesions and separation of the lesion, (C) Free nerve root.

A diagnosis of right sided intraspinal cyst at L5-S1 level with neurological compromise was made.

The patient was treated at our hospital using uniportal full endoscopic technique. Intraoperatively, the cyst appeared as nerve root and due to adhesions between dura and cyst meticulous dissection was required to identify and separate the cyst from neural structures (Fig. 2 \& Video). Patient's symptoms improved significantly after surgery and visual analogue score (VAS) of the patient was 2. At six months follow up he was faring well and asymptomatic. Histology confirmed the diagnosis of degenerative intraspinal cyst (Fig. 3). Post-operative MRI showed removal of the cyst and decompression (Fig. 4).

\section{DISCUSSION}

In the literature, various types of cysts have been described in the lumbar canal ${ }^{4,6,7)}$. These cysts are classified according to anatomical location (such as facet cysts or ligamentum flavum cysts) or pathology (such as synovial or ganglion cysts) ${ }^{1,3,7}$. Usual presentation of the spinal canal cysts are low back pain, claudication, radicular pain, motor weakness, dysasthesia all of which are secondary to the compression of the neural elements or narrowing of the canal ${ }^{2,47}$. Neuroimaging modalities like myelography, computed tomography and MRI are helpful in the diagnosis of the spinal canal cysts ${ }^{1,7)}$. Fibrous, collagenous and myxoid degeneration may be found on histological studies. In addition chronic granulomatous tissue, calcification, hemosiderin deposits may also be present ${ }^{2,47}$. Histopathology in our case confirmed degenerative intraspinal cyst.

Surgical excision followed by histopathological studies is the preferred treatment ${ }^{4,7}$. We performed the excision of the cyst with uniportal full endoscopic technique. Intraoperatively, we found a structure in right lateral recess which appeared as a nerve root and on further dissection came out to be the cyst we were looking for (Fig. 2 \& Video 1). We used full endoscopic approach in this patient because interlaminar approach endoscopic surgery has already been established as a surgical option for spinal stenosis ${ }^{5}$. For that reason we decided to excise the cyst with full endoscopic surgery but due to adhesions the task became difficult as described above. We used interlaminar PESLD (Percutaneous Endoscopic Stenosis Lumbar Decompression) method described first by Kim et al. ${ }^{5}$. With meticulous dissection using probe we confirmed that the structure
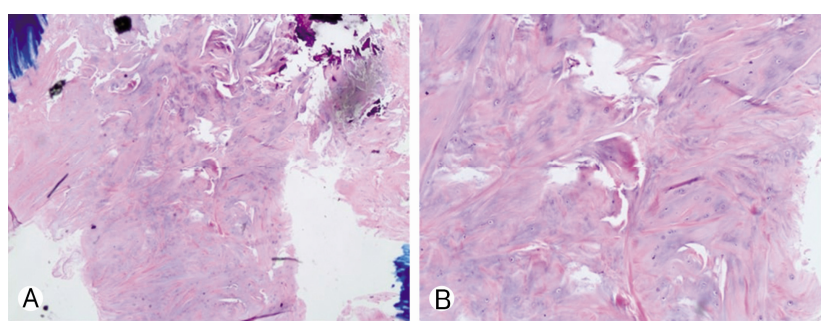

Fig. 3. Histopathology of the lesion shows Myxoid degenerative changes (A) $40 x$ and (B) $100 \times$.
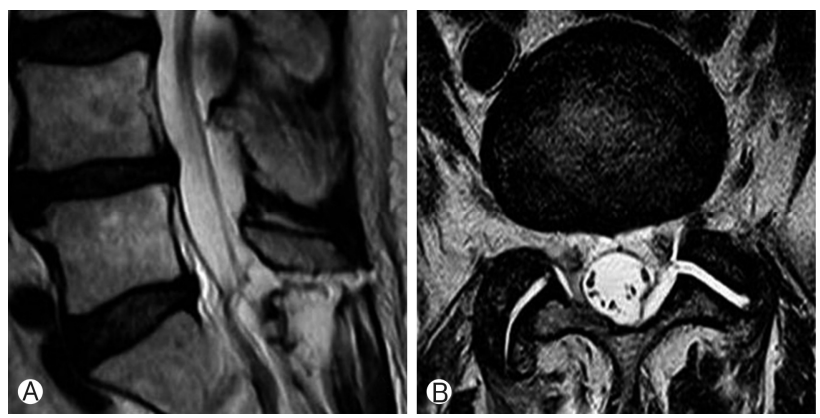

Fig. 4. Post-operative MRI showing decompression in (A) Sagittal and (B) Axial images.

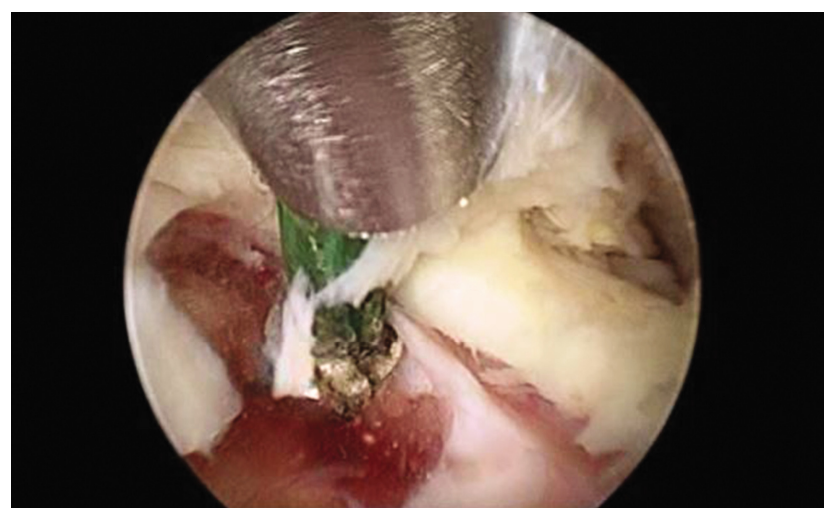

Video 1. Intraoperative video showing the lesion appearing as nerve root followed by dissection and removal.

appearing as root actually was a cyst. Careful dissection through 
adhesions was the key to success of the full endoscopic removal of the cyst.

As can be seen from this report, a cyst appearing as a nerve root is a diagnostic dilemma and surgical challenge. In endoscopic surgery utmost care is needed to recognize the neural and other vital elements lest they get violated inadvertently during the procedure.

\section{CONCLUSION}

It is not common for a cystic lesion to masquerade as nerve root. However, such a situation is a dilemma to the surgeon and poses a surgical challenge to identify the lesion correctly and save neural and other important structures. Furthermore, this case report opens another dimension to the surgical management of the intraspinal cyst with the usual advantages of the minimally invasive solution like interlaminar approach percutaneous full endoscopic lumbar stenosis lumbar decompression (PESLD).

\section{ACKNOWLEDGEMENT}

We would like to acknowledge scientific team members Ms. Jae Eun Park, Mr. Kyeong-rae Kim and Mr. Sang Hyuck Yoon for providing assistance in acquiring full text articles and managing digital works.

\section{REFERENCES}

1. Ansarib H, Khalatbaria K: MRI of degenerative cysts of the lumbar spine. Clinical Radiology 63:322-328, 2008, DOI: https://doi.org/10.1016/j.crad.2007.05.020

2. Asamoto S, Jimbo H, Fukui Y, Doi H, Sakagawa H, Ida M, Takahashi M, Shiraishi N: Cyst of the ligamentum flavumcase report. Neurol Med Chir (Tokyo) 45:653-656, 2005, DOI: https://doi.org/10.1007/s10195-010-0094-y

3. Beltagi AHE, Swamy N, Dashti F: Vacuum epidural cyst with acute neurological presentation a case report. The Neuroradiology Journal 26:213-217, 2013, DOI: https://doi.org/10.1177/197140091302600212

4. Farahmand V, Darras TN: A lumbar canal cystic mass lesion in a man with a history of chronic lower back pain. Neuolopathology 36:103-106, 2016, DOI: https://doi.org/10.1111/neup.12228

5. Kim HS, Paudel B, Jang JS, Oh SH, Lee S, Park JE, Jang IT: Percutaneous full endoscopic bilateral lumbar decompression of spinal stenosis through uniportal-contralateral approach: Techniques and preliminary results. World Neurosurg 103:201-209, 2017, DOI: http://dx.doi.org/10.1016/j.wneu.2017.03.130

6. Michael DPJ, Jeffrey SA, Eiran MM, William ZR: An instance of an atypical intraspinal cyst presenting as S1 radiculopathy: a case report and brief review of pathophysiology. Archives of Physical Medicine and Rehabilitation 85:1021-1025, 2004, https://doi.org/10.1016/j.apmr.2003.09.017

7. Taha H, Bareksei Y, Albanna W, Schirmer M: Ligamentum flavum cyst in the lumbar spine: a case report. J Orthopaed Traumatol 11: 117-122, 2010, DOI: https://doi.org/10.1007/s10195-010-0094-y 